\title{
Clinical significance of sex hormones in COVID-19
}

\author{
Nasser Mikhail* and Soma Wali \\ Department of Medicine, Endocrinology Division, Olive-View-UCLA Medical Center, David-Geffen School of Medicine, CA, USA
}

\begin{abstract}
Background: Sex hormones may play a role in excess male lethality from coronavirus disease-2019 (COVID-19).

Objective: To clarify the implications of testosterone and estradiol in the course and prognosis of COVID-19 in men and women.

Methods: PUBMED search until September 7, 2020. Search terms included: COVID-19, sex hormones, testosterone, estrogen, androgen-deprivation, mortality. Due to lack of randomized trials, we included retrospective trials, case series, and pre-print studies.

Results: In men hospitalized with COVID-19, circulating testosterone levels are generally decreased and inversely correlated with pro-inflammatory cytokines. Low testosterone levels may be associated with severe COVID-19 and high mortality. Yet, androgen deprivation therapy as treatment of prostate cancer may be associated with decreased hospitalization and favourable clinical outcomes in COVID-19. In women hospitalized with COVID-19, high testosterone levels directly correlate with pro-inflammatory cytokines. In premenopausal women, estradiol serum levels above $70 \mathrm{pg} / \mathrm{ml}$ were associated with decreased risk of having severe COVID-19. Pre-menopausal women using combined oral contraceptives may have decreased risk of COVID-19. Conversely, post-menopausal women taking hormone replacement therapy may have increased risk. In general, pregnant women with COVID-19 seem to have similar disease course as non-pregnant women, but studies were lacking adequate control subjects.
\end{abstract}

Conclusions: Preliminary data suggest that androgen deprivation therapy and estrogen could be beneficial as potential treatment of COVID-19 in men and women, respectively. However, the possible therapeutic role of these agents will be only confirmed by randomized trials.

\section{Introduction}

Accumulating data suggest that men are disproportionally affected by COVID-19 in terms of severity and mortality [1-3]. Jin et al. [1] from China were the first to report that men had more severe COVID-19 than women. They also found that death rates were 2.4 times greater in men than in women $(70.3 \%$ versus $29.7 \% ; \mathrm{P}=0.016)$ [1]. More recent data from 7 European countries and Korea showed that overall male to female ratio of mortality per 100,000 population was 1.4 [2]. The ratio varies with different ages as follows: 1.9 in the $40-49$ years age group, 2.3 in the 50-59 group, 2.6 in the 60-69 group, and 1.6 in people older than 80 years [2]. Likewise, using data from 6 countries including the USA, Green et al. [3] reported that male to female case fatality ratios were $2.53,2.92,2.57,1.83,1.57,1.58$ and 1.48 for ages $0-39,40-49,50$ $59,60-69,70-79,80-89$ and $90+$ age groups. Ding et al. [4] have shown that sex differences in COVID-19 severity and mortality may depend on menopausal status. Thus, they did not find differences in disease severity and clinical outcomes when comparing post-menopausal women with age-matched men [4]. Yet, significant differences existed between pre-menopausal women and age-matched men, with fewer pre-menopausal women suffering severe form of COVID-19 (46\% versus $75 \%$ in men, $\mathrm{P}<0.01)$, and death $(0 \%$ versus $9.7 \%$ in men, $\mathrm{P}$ $<0.01$ ) [4]. These results suggest that pre-menopausal state may have protective effect against COVID-19. Most researchers believe that excess mortality in men relative to women is largely due to higher prevalence of smoking, cardiovascular and lung disease co-morbidities [5]. Using a multinational registry formed of 14,712 patients with COVID-19, Alkhouli et al. [5] found that all-cause mortality was $8.8 \%$ in men and $4.3 \%$ in women, odds ratio (OR) $2.15,95 \%$ CI, $1.87-2.46$; $\mathrm{P}<0.01)$. After propensity score matching to control for differences in age and co-morbidities, all-cause mortality was slightly attenuated but remained significantly higher in men than in women, $8.13 \%$ versus 4.60\%, OR 1.81, 95\% CI, 1.55-2.11; P < 0.001) [5]. Therefore, there are factors beyond co-morbidities that contribute to excess mortality in men with COVID-19. These factors may include gender differences in immune response to infections and sex hormones [6,7]. This article will focus on the possible implication of sex hormones in the clinical course of COVID-19 in men and women based on available studies.

\section{Relevance of sex hormones to COVID-19}

There are 2 opposing theories regarding the role of testosterone in COVID-19 [8]. First, low testosterone levels can cause a reduction of respiratory muscle activity and testosterone therapy improve peak oxygen consumption and functional exercise capacity [9]. In addition, testosterone has anti-inflammatory effect associated with reduction in pro-inflammatory cytokines such as interleukin- 6 (IL-6) and interferon- $\alpha$ (IFN- $\alpha)$ as well as markers of severe inflammation such as C-reactive protein $[7,10]$. This anti-inflammatory effect could virtually decrease severity of cytokine storm, the main cause of acute respiratory distress syndrome (ARDS) and mortality in COVID-19. On the other hand, the severe acute respiratory syndrome coronavirus 2 (SARSCov-2) uses the angiotensin converting enzyme 2 (ACE2) as receptor

${ }^{\star}$ Correspondence to: Nasser Mikhail, Department of Medicine, Endocrinology Division, Olive-View-UCLA Medical Center, David-Geffen School of Medicine, CA, USA, Tel: 1 747-210-3205; E-mail: nmikhail@dhs.lacounty.gov

Key words: COVID-19, testosterone, estrogen, inflammatory markers, androgen deprivation therapy

Received: April 03, 2020; Accepted: April 20, 2020; Published: April 24, 2020 
and the transmembrane protease serine 2 (TMPRSS2) as co-receptor for host cell binding and penetration [11]. Testosterone is known to upregulate TMPRSS2, and therefore testosterone could potentially facilitate SARS-Cov-2 cell binding and spread [8]. As far as estrogen is concerned, this hormone is believed to play a protective role against COVID-19 in large part due to its anti-inflammatory effect, analogous to that of testosterone [7]. In the following sections, the authors will try to elucidate the implications of sex hormones in men and women with COVID-19 based on available data.

\section{Testosterone in patients with COVID-19}

\section{Male studies}

In one Chinese retrospective study, Ma et al. [12] compared serum levels of testosterone in 81 young men (median age 38 years) hospitalized for COVID-19 (86\% of them had moderate disease) with 100 healthy control subjects. They found a non-significant trend of testosterone levels to be lower in COVID-19 patients compared with control subjects, median (interquartile range) being 397 (312-568) and 479 (349-562) ng/dl, respectively ( $\mathrm{P}=0.094)$ [12]. Meanwhile luteinizing hormone $(\mathrm{LH})$ values were significantly higher in patients compared with control individuals; median 5.9 (4.3-8.2) and 3.2 (2.5-4.6) $\mathrm{mU} / \mathrm{L}$, respectively, $\mathrm{P}<0.0001)$ [12]. It follows that the testosterone/ $\mathrm{LH}$ ratio, a marker of testicular function, was significantly lower in patients with COVID-19 compared with healthy subjects [12]. This observation suggests some degree of testicular dysfunction in men with COVID-19. Moreover, plasma levels of C-reactive protein, an index of inflammatory severity in COVID-19, were negatively associated with testosterone/LH ratio [12]. In another retrospective study from Germany, Schroeder et al. [13] measured testosterone in 35 critically ill men (median age 62 years, range 31-80) with COVID-19 admitted to the intensive care unit (ICU). They found that majority of patients $(68 \%)$ had low testosterone levels, and $54 \%$ of them had very low testosterone values below $141 \mathrm{ng} / \mathrm{dl}$ (normal range in 50 years-old men $192-743 \mathrm{ng} / \mathrm{dl}$ [13]. In addition, $32 \%$ of all men and $17 \%$ of men with low testosterone had elevated LH consistent with testicular dysfunction [13]. Like the study of Ma et al. [12], Schroeder et al. [13] reported that low testosterone levels correlated with inflammatory cytokines: IL-2, and IFN- $\alpha$. Furthermore, Schroeder et al. [13] observed that male patients with low testosterone appeared to have increased mortality risk compared with those with normal testosterone levels. However, the magnitude of this increased risk was not mentioned [13]. In agreement with the prior 2 studies, Rastrelli et al. [14] found that total testosterone levels were negatively associated with several indices of COVID-19 severity, namely neutrophil count, lactate dehydrogenase (LDH) and ferritin. On the other hand, using data from UK Biobank, Ghazizadeh et al. [15] showed significant direct association between free androgen index $[=100 \mathrm{x}$ total testosterone $(\mathrm{nmol} / \mathrm{L}) /$ sex-hormone binding globulin (nmol/L)] and susceptibility and severity of COVID-19. Thus, for each standard deviation (SD) increase in free androgen index there was increased risk of positive COVID-19 test (OR 1.22, 95\% CI 1.03$1.45 ; \mathrm{P}=0.024)$, as well as risk of severe COVID-19 infection $(\mathrm{OR}=1.22$, 95\% CI 1.02-1.46; $\mathrm{P}=0.031)]$. [15

Taken together, most [12-14] but not all [15] studies, suggest that low testosterone concentrations in men hospitalized with COVID-19 may be associated with a more severe form of COVID-19. The mechanisms of the latter observation are not fully understood. The fact low testosterone levels are associated in many patients with concomitant elevation in $\mathrm{LH}$ values points to some degree of testicular failure (i.e. hypergonadotropic hypogonadism). It is possible that
SARS-Cov-2 may directly invade the testicles. In fact, Wang et al. [16] have shown that ACE2, the receptors of SARS-Cov-2 as mentioned earlier, are present in several testicular tissues including Leydig cells (producing testosterone), Sertoli cells (producing inhibin B), and in spermatogonia (the sperm-producing cells). However, to the best of authors' knowledge, no cases of orchitis have been reported in relation to COVID-19. A second possibility is that hypogonadism is common in patients with COVID-19 as result of co-morbidities such as diabetes, obesity, obstructive sleep apnea [17]. A third possibility is that low testosterone levels may be a marker of severe illness in hospitalized patients in general irrespective of underlying diseases. In one prospective study of 150 elderly men (mean age 86 years, range 68-99) admitted to the hospital with various diseases, hypogonadism was a strong independent predictor of in-hospital and long-term (up to 5 years) total mortality (adjusted hazard ratio 3.35, 95\% CI 1.53-7.23; $\mathrm{P}=0.0002$ ) and cardiovascular mortality (adjusted hazard ratio 2.14, 95\% CI 1.18-3.86; P <0.05) [18].

\section{Female studies}

Contrary to their investigations in men with COVID-19, Schroeder et al. [13] found that 6 of 10 hospitalized female patients with COVID-19 (median age 67 years, range 54-84) had elevated serum testosterone above $46 \mathrm{ng} / \mathrm{dl}$ (normal post-menopausal testosterone 2.6-43.2 ng/dl). Moreover, high, not low, testosterone levels correlated with inflammatory cytokines in women [13]. Ghazizadeh et al. [15] failed to find significant association between free androgen index and COVID-19 susceptibility and severity in their female patients with COVID-19. Clearly, the relationship between testosterone and COVID-19 in women deserves further studies.

\section{Testosterone and COVID-19 mortality in men}

In a retrospective Italian study, Rastrelli et al. [14] evaluated the relationship of testosterone levels to clinical outcomes in 31 men admitted to the "respiratory" ICU with COVID-19. Median serum testosterone concentrations were significantly lower in the 4 patients whose clinical condition deteriorated and were either transferred to ICU or died compared with patients whose condition improved, 28 and $253 \mathrm{ng} / \mathrm{dl}$, respectively [14]. In addition, compared with patients who did not develop ARDS, patients with COVID-19 complicated by ARDS had lower testosterone levels (63 versus $201 \mathrm{ng} / \mathrm{dl}$ ) and higher LH levels (12.0 versus $6.9 \mathrm{mU} / \mathrm{L}$ ) [14]. Moreover, these authors showed that for each $28.8 \mathrm{ng} / \mathrm{dl}(1 \mathrm{nmol} / \mathrm{L})$ decrease in circulating testosterone concentrations, the probability of having worse outcome (ICU transfer or death) increased; OR 1.42, 95\% CI 1.06-1.89; $\mathrm{P}=0.027$ ) [14]. They further defined a testosterone threshold level of $144 \mathrm{ng} / \mathrm{dl}$, below which probability of ICU transfer or death increased 10 times [14]. The results of this study are in concordance with previous investigations showing positive correlation between low testosterone levels and increase inflammatory cytokines in men with COVID-19 [12,13].

\section{Androgen deprivation therapy and COVID-19 mortality in men}

As mentioned earlier, there is a theory that testosterone may enhance COVID-19 infection by increasing synthesis of the co-receptor of SARS-Cov-2: TMPRSS2 [8]. Therefore, by lowering androgen levels, androgen deprivation therapy (ADT) could potentially inhibit virus cellular binding and subsequent infection. Two retrospective studies suggested that ADT may decrease susceptibility and severity of COVID-19. Montopoli et al. [19] reported that only 4 of 5273 (0.07\%) patients with prostate cancer treated with ADT had COVID-19. 
Furthermore, they found that these 4 patients had approximately 4 times lower risk of having COVID-19 compared with patients who did not receive ADT [19]. In addition, none of the 4 patients have died from COVID-19 [19]. In another preliminary report from New York, Patel et al. [20] found that compared with no use of ADT, use of ADT in 22 patients with prostate cancer was associated with lower rates of hospitalization (OR $0.26,95 \%$ CI $0.06-0.79 ; \mathrm{P}<0.02$ ) and requirements for supplemental oxygen (OR 0.26, 95\% CI 0.07-0.92; P 0.026) [20]. Additionally, there was a trend towards lower mortality and need for intubation on the group of patients on ADT [20]. Taken together, the results of these 2 studies suggest that achievement of extremely low testosterone levels by ADT may confer a protective effect against COVID-19 $[19,20]$. Meanwhile, a third study from Italy did not support the latter concept. Caffo et al. [21] reported that 36 of 1949 (1.8\%) with prostate cancer had COVID-19. Eleven of these 36 (30.6\%) patients died from COVID-19 [21]. This lethality rate was similar and not lower than that of Italian men with similar age infected with COVID-19 [21]. Nevertheless, the increased mortality reported by Caffo et al. [21] may be partly due to the fact that their patients had advanced metastatic prostate cancer.

\section{Estrogen in patients with COVID-19}

\section{Male studies}

In the series of 81 young men (median age 38 years) hospitalized with COVID-19 reported by Ma et al. [12], estradiol levels (the main estrogen) were similar to those of healthy men (median $32 \mathrm{pg} / \mathrm{ml}$ ). Shroeder et al. [13] found that $47 \%$ of men (median age 62 years) had elevated estradiol levels between $52-185 \mathrm{pg} / \mathrm{ml}$ (normal male range 27.1-52.2 pg/ml). They also found a positive correlation between estradiol levels and the pro-inflammatory cytokine IL-6 [13].

\section{Female studies}

In 78 pre-menopausal women hospitalized for COVID-19, Ding et al. [4] found that estradiol levels greater than $70 \mathrm{pg} / \mathrm{ml}$ were associated with decreased risk of having severe COVID-19, hazard ratio $0.30(95 \%$ CI 0.09-1.001; $\mathrm{P}=0.05)$ after adjustment for age, phase of menstrual cycle and co-morbidities. In addition, there was inverse correlation between estradiol levels and the cytokines IL-6, IL-8 and TNF- $\alpha$ [4]. Hence, these results are consistent with estrogen protective effect in COVID-19. In a study from UK called The COVID Symptom Study Smartphone Application "app", Costeira et al. [22] reported that postmenopausal women had a higher rate of predicted COVID-19 compared with pre-menopausal women (OR 1.22, 95\% CI 1.07-1.39; $\mathrm{P}=0.03$ ) [22]. Likewise, women using combined oral contraceptives (COCP) had a lower rate of predicted COVID-19 (OR=0.87, 95\% CI 0.64 0.97; $\mathrm{P}=0.023$ ) compared with age-matched women not taking COCP [22]. Conversely, women aged 50-65 years using post-menopausal hormone replacement therapy had higher predicted COVID-19 than age-matched women not taking such therapy $(\mathrm{OR}=1.32$, 95\% CI 1.161.49) [22]. The authors mentioned that co-morbidities, duration and use of postmenopausal hormone therapy might explain the unexpected increase in predicted COVID-19 in this group of post-menopausal women [22].

\section{COVID-19 in pregnancy}

Since pregnancy is characterized by extremely high levels of circulating estrogen, evaluation of susceptibility and clinical severity of COVID-19 in pregnant women may be a useful tool to examine the possible protective effect of estrogen. In general, available data suggest that severity of COVID-19 in pregnant women may not be different from non-pregnant women $[23,24]$. This notion is limited, however, by the lack of direct comparison with age-matched non-pregnant women with similar co-morbidities. A possible protective effect of estrogen was suggested in the study of Chen et al. [23] including 118 pregnant women hospitalized for COVID-19. In this study, 6 of the 9 pregnant women whose condition deteriorated did so following delivery when serum estrogen levels declined.

\section{Conclusions and current directions}

Our knowledge regarding sex hormones and COVID-19 is still in its stage of infancy. In men, low testosterone levels are generally associated with high inflammatory markers and disease severity. Whether low testosterone in men is a cause or a consequence (or both) in COVID-19 is still unclear. The preliminary observations of favorable prognosis of COVID-19 in men taking ADT supports the theory that low testosterone levels may be beneficial in COVID-19. This issue will be clarified by results of ongoing trials that compare outcomes of patients with COVID-19 randomized to ADT versus placebo. In women, preliminary data suggests that estrogen may have a beneficial effect in COVID-19. This is based on the following observations: premenopausal state is generally protective against COVID-19, higher serum estradiol levels are associated with less severe COVID-19, and decreased risk of COVID-19 in pre-menopausal using COCP. However, the role of estrogen will be only clarified by randomized trials. Such trials are underway to evaluate efficacy and safety of estrogen for treatment of COVID-19 in both women and men.

\section{References}

1. Jin JM, Bai P, He W, Wu F, Liu XF, et al. (2020) Gender Differences in Patients With COVID-19: Focus on Severity and Mortality. Front Public Health 8: 152. [Crossref]

2. Bhopal SS, Bhopal R (2020) Sex differential in COVID-19 mortality varies markedly by age. Lancet 396 : $532-533$.

3. Green MS, Swartz N, Nitzan D, Peer V (2020) The male excess in case-fatality rates for COVID-19. A meta-analytic study of the age-related differences and consistency over six countries. MedRxiv.

4. Ding T, Zhang J, Wang T, Cui F, Chen Z, et al. (2020) Potential Influence of Menstrual Status and Sex Hormones on female SARS-CoV-2 Infection: A Cross-sectional Study from Multicentre in Wuhan, China. Clin Infect Dis: ciaa1022. [Crossref]

5. Alkhouli M, Nanjundappa A, Annie F, Bates MC, Bhatt DL (2020) Sex Differences in Case Fatality Rate of COVID-19: Insights From a Multinational Registry. Mayo Clin Proc 95: 1613-1620. [Crossref]

6. Klein SL, Flanagan KL (2016) Sex differences in immune responses. Nat Rev Immunol 16: 626-638. [Crossref]

7. Al-Lami RA, Urban RJ, Volpi E, Algburi AMA, Baillargeon J (2020) Sex Hormones and Novel Corona Virus Infectious Disease (COVID-19). Mayo Clin Proc 95: 17101714.

8. Pozzilli P, Lenzi A (2020) Commentary: Testosterone, a key hormone in the context of COVID-19 pandemic. Metabolism 108: 154252. [Crossref]

9. Caminiti G, Volterrani M, Iellamo F, Marazzi G, Massaro R, et al. (2009) Effect of long-acting testosterone treatment on functional exercise capacity, skeletal muscle performance, insulin resistance, and baroreflex sensitivity in elderly patients with chronic heart failure a double-blind, placebo-controlled, randomized study. $J$ Am Coll Cardiol 54: 919-927. [Crossref]

10. Bianchi VE (2018) The Anti-Inflammatory Effects of Testosterone. J Endocr Soc 3 : 91-107. [Crossref]

11. Hoffmann M, Kleine-Weber H, Schroeder S, Krüger N, Herrler T, et al. (2020) SARSCoV-2 Cell Entry Depends on ACE2 and TMPRSS2 and Is Blocked by a Clinically Proven Protease Inhibitor. Cell 181: 271-280.e8. [Crossref]

12. Ma L, Xie W, Li D, Shi L, Ye G, et al. (2020) Evaluation of sex-related hormones and semen characteristics in reproductive-aged male COVID-19 patients [published online ahead of print, $2020 \mathrm{Jul}$ 4]. J Med Virol: 10.1002/jmv.26259. [Crossref] 
13. Schroeder M, Tuku B, Jarczak D, Nierhaus A, Bai T, et al. (2020) The majority of male patients with COVID-19 present low testosterone levels on admission to Intensive Care in Hamburg, Germany: a retrospective cohort study. MedRxiv.

14. Rastrelli G, Di Stasi V, Inglese F, Beccaria M, Garuti M, et al. (2020) Low testosterone levels predict clinical adverse outcomes in SARS-CoV-2 pneumonia patients. Andrology: 10.1111/andr.12821. [Crossref]

15. Ghazizadeh Z, Majd H, Richter M, Samuel R, Zekavat SM, et al. (2020) Androgen Regulates SARS-CoV-2 Receptor Levels and Is Associated with Severe COVID-19 Symptoms in Men. Preprint. bioRxiv.

16. Wang Z, Xu X (2020) scRNA-seq Profiling of Human Testes Reveals the Presence of the ACE2 Receptor, A Target for SARS-CoV-2 Infection in Spermatogonia, Leydig and Sertoli Cells. Cells 9: 920. [Crossref]

17. Bhasin S, Brito JP, Cunningham GR, Hayes FJ, Hodis HN, et al. (2018) Testosterone Therapy in Men with Hypogonadism: An Endocrine Society Clinical Practice Guideline. J Clin Endocrinol Metab 103: 1715-1744. [Crossref]

18. Iglesias P, Prado F, Ridruejo E, Muñoz A, Macías MC, et al. (2015) Hypogonadism and Mortality in Aged Hospitalized Male Patients: A 5-Year Prospective Observational Study. Exp Clin Endocrinol Diabetes 123: 589-593. [Crossref]
19. Montopoli M, Zumerle S, Vettor R, Rugge M, Zorzi M, et al. (2020) Androgendeprivation therapies for prostate cancer and risk of infection by SARS-CoV-2: a population-based study $(\mathrm{N}=4532)$. Ann Oncol 31: 1040-1045. [Crossref]

20. Patel VG, Zhong X, Liaw B, Tremblay D, Tsao CK, et al. (2020) Does androgen deprivation therapy protect against severe complications from COVID-19? Ann Oncol 31: 1419-1420. [Crossref]

21. Caffo O, Zagonel V, Baldessari C, Berruti A, Bortolus R, et al. (2020) On the relationship between androgen-deprivation therapy for prostate cancer and risk of infection by SARS-CoV-2. Ann Oncol 31: 1415-1416. [Crossref]

22. Costeira R, Lee KA, Murray B, Christiansen C, Castillo-Fernandez J, et al. (2020) Estrogen and COVID-19 symptoms: associations in women from the COVID Symptom Study. MedRxiv.

23. Chen L, Li Q, Zheng D, Jiang H, Wei Y, et al. (2020) Clinical Characteristics of Pregnant Women with Covid-19 in Wuhan, China. N Engl J Med 382: e100.

24. Ryan GA, Purandare NC, McAuliffe FM, Hod M, Purandare CN (2020) Clinical update on COVID-19 in pregnancy: A review article. J Obstet Gynaecol Res 46: 1235-1245.

Copyright: $\odot 2020$ Mikhail N. This is an open-access article distributed under the terms of the Creative Commons Attribution License, which permits unrestricted use, distribution, and reproduction in any medium, provided the original author and source are credited. 